\title{
Deepening our relation-in-practice
}

\author{
Debbie MacLellan and Jacqui Gingras, JCD Co-Editors
}

Following the success of our IIth International World Dietetics conference, with the theme "lt's High Time: From Awareness to Action: Advancing social justice through critical health pedagogies," we are continuing to see more submissions to the Journal that contend with social justice and equity concerns. In this issue of the Journal of Critical Dietetics, we present articles that assert meaningful critique regarding food security, inclusion, whiteness, food policy, and eating disorders. These topics, and the means by which they are contextualized, are received like a cool glass of water on a stifling hot day; much needed and ultimately refreshing.

Despite intending to publish articles as they arrived, we found ourselves with many articles arriving in close proximity, so once again, we are publishing an entire issue. It is a feast for the equity senses. This issue includes several firsts for the Journal; we have our first letter to the editor and a response. Kate Burt's article on the whiteness of the Mediterranean diet from Volume 5, Issue 2, motivated a response from Kelly Toups, to which Kate Burt again responded. This is one of the foundational activities of critical scholarship; a public sharing, responding, and engagement with each other's work. We are pleased to see that happening among the scholarly community through our Journal and encourage others to engage in this form of critical inquiry.

As always, we have a wide array of research articles starting with DiBiasse and colleagues' work on experiences of LGBTQ-identifying students, interns, and practitioners of dietetics. The second first is the publication of the survey that DiBiasse and colleagues used in their project. This survey is available for all to use as long as correct attribution is given. This is a crucial aspect of the open source movement; sharing not only the outputs of research, but the tools by which data was gathered. We present that to you without hiding it behind a paywall and with the authors' permission.

Next, Baxter and colleagues present a well-documented argument for improved demographic data in the nutrition and dietetics profession in the United States in order to more accurately assess race/ethnic and gender diversity. This is followed by an article by Fuster, Leak, and Galitzdorfer who examined dietitians' experiences working with Hispanic Caribbean communities in New York City, thus expanding our understanding of cultural competence in dietetic practice.

Two research teams investigated issues related to food insecurity in Ontario, Canada. Spoel and Derkatch examined the values of food security and food literacy in Ontario's Food Charter Toolkits, unearthing an uneasy relationship between the two. Belyea and colleagues analyzed how research using the Nutritious Food Basket protocol is presented and mobilized on the public health websites in Ontario, suggesting that these websites are not used as effectively as they could be for promoting food security advocacy activities.

Our final two research studies in this issue explored problems related to the practice of dietetics in an eating disorder specialty. Bradshaw, Caron, and Brown identified potential barriers to specializing in eating disorder treatment and compared barriers experienced by dietitians who self-identified as white and those from underrepresented racial/ 
ethnic groups. Setnick and colleagues surveyed dietitians working with clients with disordered eating to determine education and training needs and implications for clinical practice.

The final insight article in this issue was produced from a roundtable discussion about public health in Mexico and beyond. This panel presentation was part of the 202I Just Food conference and should be of interest to anyone working on or thinking about health and its representation in Mexico and beyond.

We know how challenging these past two years have been for everyone and want to thank our authors, reviewers, and readers for your ongoing support for the Journal of Critical Dietetics. Our hope is that you will continue to find inspiration in our Journal and to submit, review, read, and share articles that contribute to meaningful discussions about critical dietetics issues. 